\title{
Regular self-monitoring of blood glucose needed for metabolic control
}

Regular self-monitoring of blood glucose (SMBG) helps to achieve lower levels of $\mathrm{HbA}_{1 \mathrm{c}}$, an indicator of good metabolic control, in children and adolescents with type 1 diabetes mellitus (T1DM), according to a German research group.

"In children with diabetes mellitus, it is sometimes difficult to convince them that regular SMBG is of great importance and value to their continuous treatment," explains lead researcher Ralph Ziegler (Clinic for Pediatric and Adolescent Diabetes, Münster, Germany). However, little is known about the relationship between SMBG frequency and treatment quality.

The researchers analyzed data from 26,723 children (aged $0-18$ years) with T1DM. The data were recorded in the DPV-Wiss database of diabetes mellitus care and clinical outcomes in Germany and Austria during 1995-2006.

Metabolic control improved when SMBG was performed two to five times per day. This relationship was strongest in those $>12$ years old. However, increasing the frequency of SMBG above five times a day did not further improve metabolic control.

The investigators speculate that frequent SMBG enables patients to effectively modify their diabetes mellitus management, which results in improved metabolic control. This effect might not have been found in those $<12$ years old as increased parental involvement in the management of diabetes mellitus in young children results in a stricter regimen than in teenagers.

Regular SMBG is necessary to achieve good metabolic control in children and adolescents and to avoid diabetic complications, conclude the researchers.

\section{Claire Greenhill}

Original article Ziegler, R. et al. Frequency of SMBG correlates with $\mathrm{HbA}_{1 \mathrm{c}}$ and acute complications in children and adolescents with type 1 diabetes. Pediatr. Diabetes doi:10.1111/j.1399-5448.2010.00650.x 